\title{
The Impact of the COVID-19 Pandemic on Children's Conduct Problems and Callous-Unemotional Traits
}

\author{
Rebecca Waller ${ }^{1} \cdot$ Tralucia Powell $^{2} \cdot$ Yuheiry Rodriguez $^{1} \cdot$ Natalie Corbett $^{1} \cdot$ Samantha Perlstein $^{1}$. \\ Lauren K. White ${ }^{3,4,5} \cdot$ Ran Barzilay ${ }^{3,4,5} \cdot$ Nicholas J. Wagner $^{2}$ (1)
}

Accepted: 5 December 2020 / Published online: 6 January 2021

(c) The Author(s), under exclusive licence to Springer Science+Business Media, LLC part of Springer Nature 2021

\begin{abstract}
The COVID-19 pandemic has dramatically altered family life, but whether family exposures to and worries about the COVID-19 pandemic has impacted child conduct problems (CP) and callous-unemotional (CU) traits is unknown. Thus, we evaluated 303 parents $\left(M_{\text {age }}=38.04 ; \mathrm{SD}=5.21 ; 92.4 \%\right.$ biological mothers $)$ and children $\left(M_{\text {age }}=6.43 ; \mathrm{SD}=2.13\right.$; $51.8 \%$ female) during a four-month period early in the pandemic. We examined associations between parental exposures to COVID-19, parental worries about the pandemic, harsh and warm parenting practices, and child CP and CU traits. Although more parental worries were not directly related to parenting practices, more worry about COVID-19 was specifically related to higher levels of child CP, particularly parental worries about themselves or family members contracting the virus. Our findings add to a growing literature demonstrating the burden that the pandemic has placed on families and its implications for children's mental health.
\end{abstract}

Keywords Callous-unemotional traits $\cdot$ Conduct problems $\cdot$ Families $\cdot$ Parenting $\cdot$ Prevention science

\section{Background}

Childhood conduct problems (CP) increase risk for psychiatric disorders across the lifespan and are associated with costly and adverse educational and health outcomes [1,2]. Callous-unemotional (CU) traits are defined by deficient

Supplementary Information The online version of this article (https://doi.org/10.1007/s10578-020-01109-y) contains supplementary material, which is available to authorized users.

Nicholas J. Wagner

njwagner@bu.edu

1 Department of Psychology, University of Pennsylvania, Philadelphia, PA 19104, USA

2 Department of Psychological and Brain Sciences, Boston University, 64 Cummington Mall, Boston, MA 02215, USA

3 Department of Psychiatry, Perelman School of Medicine, University of Pennsylvania, Philadelphia, PA 19104, USA

4 Lifespan Brain Institute, Children's Hospital of Philadelphia and Penn Medicine, University of Pennsylvania, Philadelphia, PA 19104, USA

5 Department of Child and Adolescent Psychiatry and Behavioral Sciences, Children's Hospital of Philadelphia, Philadelphia, PA 19104, USA empathy, lack of guilt, and poor moral regulation [3], and predict early-starting and severe trajectories of $\mathrm{CP}$ across childhood [4-6] and adolescence [7, 8]. CU traits were added to the DSM-5 as a specifier for the diagnosis of conduct disorder [9], emphasizing the importance of understanding the etiology of $\mathrm{CU}$ traits to inform personalized treatments for CP [10]. Despite genetic influences on CP [11], multiple aspects of caregiving inadvertently socialize children to become aggressive, non-compliant, or violent $[12,13]$. In particular, harsh and intrusive parenting contribute to cycles of coercive parent-child interactions [14, 15], whereas positive reinforcement (e.g., contingent use of praise) and proactive parenting practices (e.g., scaffolding) predict reductions in childhood CP [16, 17]

Early studies concluded that CU traits developed independently of parenting [18]. Twin studies appeared to support this conclusion with $40 \%-78 \%$ of the variance in CU traits attributable to genetic influences [19]. Indeed, children with $\mathrm{CU}$ traits show inherited characteristics that make parenting more challenging and increase risk for $\mathrm{CP}$, including punishment insensitivity and low social affiliation [20, 21]. However, recent research has begun to establish specific parenting factors that predict $\mathrm{CU}$ traits, work that is paramount for informing personalized treatments for $\mathrm{CP}$, even in 
the context of heritable risk [10]. Several studies, including those that have used genetically-informed designs, suggest that parental harshness (i.e., physically or verbally punitive) is a general risk factor for both $\mathrm{CP}$ and $\mathrm{CU}$ traits. The experience of harshness desensitizes children to cues of threat and models aggression as an effective interpersonal strategy $[22,23]$. Low parental warmth (i.e., lack of affection and nurturance) is thought to specifically exacerbate risk for $\mathrm{CU}$ traits. Low warmth restricts the experience of affiliative parent-child interactions and stymies positive reinforcement, thus limiting children's opportunities to enact or experience empathy, caring, or cooperation, which increases their risk for developing CU traits [23-25]. This conclusion is supported by recent treatment studies where adjunctive modules for parenting interventions focused on emotion engagement and increasing parental warmth lead to reductions in $\mathrm{CU}$ traits $[26,27]$.

At the same time, we know that maladaptive child emotional and behavioral responses, including the development of $\mathrm{CP}$ and $\mathrm{CU}$ traits, are subject to many individual and contextual factors beyond the sole influence of parenting experiences [28-30]. The bioecological [31] and family stress models [32] illustrate how multiple aspects of children's environments, including living in poverty, neighborhood dangerousness, and daily home stressors, have a cascading impact on development [33]. For example, there are established links between low family income and risk for CP, evidenced through both quasi-experimental [34] and experimental [35] study designs. Similarly, a meta-analysis demonstrated links between greater family stress and risk for $\mathrm{CP}$ and CU traits [36], and longitudinal research has linked greater family disorganization in the first three years of life with later CU traits [29]. Moreover, greater parental stress or worry may indirectly contribute to children's $\mathrm{CP}$ or CU traits via parenting behaviors, including fewer supportive emotion vocalizations [37], less empathic parenting [38], and greater harshness [39]. Together, this prior literature suggests that environmental stressors that impact both the home and community can act as both direct and indirect risk factors for children's psychopathology, including CP and $\mathrm{CU}$ traits.

Since the beginning of 2020, the COVID-19 pandemic has thrust an additional host of stressors onto parents and families, with broad implications for child development and mental health [40]. Beyond the colossal impact of COVID19 on the general health and well-being of society, many parents have had to simultaneously manage the full-time care and education of children while keeping a job or working from home [41, 42]. At the same time, families have had to adopt physical distancing, eliminating many social support or leisure activities that would otherwise promote positive parent-child interactions [43]. These circumstances are concerning in light of robust evidence linking family stress and low parental social support to increased risk for harsh and abusive parenting [40,44], with concomitant effects on CP and CU traits [29, 45]. In particular, several commentaries published at the start of the pandemic raised the alarm that the deleterious combination of factors associated with the pandemic may lead to an increased risk for child abuse and an escalation in violence exposure, which could impact childhood CP and CU traits [40, 43]. Recent work by Brown and colleagues (2020) demonstrates that the COVID-19 pandemic has contributed to increases in parents' perceived stress and subsequent risk for child abuse [46], with important implications for the current study when considered alongside extant research findings linking parental stress both to CP [39] and CU traits [47].

Moreover, the lives of children have been directly negatively impacted by the pandemic through home confinement, school closures, limited social interactions with peers, and stifled access to playgrounds and team sports [40, 41, 48]. Even at young ages, children are also cognizant of the threats posed by illness and death $[49,50]$ and sensitive to family stress [32]. While the direct, detrimental effects of pandemics on children are not well-documented, increases in posttraumatic stress disorder (PTSD) symptoms were reported among children isolated because of the $\mathrm{H} 1 \mathrm{~N} 1$ pandemic [51] and increases in aggression and PTSD symptoms were reported among children exposed to natural disasters [52, 53]. However, no studies have explored associations between family exposure to and worries about COVID-19 and child $\mathrm{CP}$ and $\mathrm{CU}$ traits, and whether the influence of the pandemic on children persists after accounting for the known and robust effects of the parental caregiving environment. This knowledge can help to further inform our understanding of how chronic environmental stress can impact children's CP and $\mathrm{CU}$ traits and guide future family-based interventions to support parents and children dealing with the long-term mental health consequences of the pandemic.

To advance knowledge about how the direct negative sequalae of COVID-19 and parenting practices during the pandemic impact $\mathrm{CP}$ and $\mathrm{CU}$ traits, the current study evaluated a sample of parents and children in the early Spring and Summer of 2020, encompassing a period with high community transmission and stay-at-home orders due to COVID-19. First, we examined associations between family characteristics and exposure to (e.g., job loss, knowing people who died from COVID-19) and worries about COVID-19 (e.g., contracting the virus, the financial burden of the pandemic). Second, we sought to confirm that established links between parenting and $\mathrm{CP}$ and $\mathrm{CU}$ traits persist in the context of heightened familial stress. That is, we tested whether hypothesized associations between higher parental harshness and CP and CU traits, and between lower parental warmth and child CU traits would manifest during this period of enhanced and pervasive stress facing families. 
Third, we tested whether parental COVID-19 exposures and worries were related to their parenting behaviors. Finally, we examined whether parent reports of risk exposures to and worries about COVID-19 directly impacted children's CP and CU traits, over and above the impact of harsh and warm parenting practices. We hypothesized that the burden facing families in the form of financial and health concerns would directly affect children's mental health well-being, beyond their parental caregiving environment.

\section{Methods}

\section{Participants}

Participants were 303 children were aged 3.00 to 10.94 years old $\left(M_{\text {age }}=6.43 ; \mathrm{SD}=2.13 ; 51.8 \%\right.$ female $)$, recruited from two cities in the northeastern United States (US) ([blind for review], $n=160$; [blind for review], $n=143$ ). All data were collected from parents of those children (biological mothers $=92.4 \%$; biological fathers $=2.0 \%$; foster or adoptive mother $=5.0 \%$; other female caregiver $=0.7 \%$ ) who reported being from the following racial groups: White $(n$ $=224 ; 73.9 \%)$, Black/African-American $(n=36 ; 11.9 \%$, including $n=4$ biracial), Asian ( $n=30 ; 9.9 \%$, including $n=5$ biracial), and other $(n=13 ; 4.3 \%)$. Children $(51.4 \%$ female) were from the following racial groups: White $(n$ $=199 ; 65.7 \%)$, Black/African-American $(n=49 ; 16.2 \%$, including $n=13$ biracial $)$, Asian $(n=37 ; 12.2 \%$, including $n=22$ biracial), and other $(n=18 ; 5.9 \%)$. In terms of ethnicity, 13 parents $(4.3 \%)$ and 31 children $(10.2 \%)$ were reported to be Hispanic/Latino/a. the families in this study $60.5 \%$ of parents reported having a graduate-level degree, 24.4\% had a Bachelor level degree, $4.7 \%$ had an Associates level qualification, and $10.4 \%$ had a high school qualification or less. The average monthly household income ranged from $\$ 302$ to $\$ 75,000(M=\$ 11,516, \mathrm{SD}=\$ 8,205)$. Parents reported on whether children had previously been diagnosed with an internalizing disorder, including depression or anxiety $(n=9,3 \%)$ or an externalizing disorder, such as oppositional defiant or attention-deficit hyperactivity disorder $(n=$ 17, 5.6\%). An overlapping subset of children had Individual Education Plans (IEPs; $n=17,5.6 \%$ ), were taking psychiatric medications $(n=13,4.3 \%)$, or were receiving behavioral therapy $(n=10,3.3 \%)$.

\section{Procedures}

Participants were recruited in April $(n=35)$, May $(n=101)$, June ( $n=73)$, and July $(n=94)$ of 2020 via Facebook ads with the geographic location restricted to the wider metropolitan areas of [blind for review] and [blind for review], flyers posted in community locations in these cities (e.g., daycares, public playgrounds, and grocery stores), and targeted recruitment through institutionally-maintained databases of families interested in participating in research. Interested families were directed to an online form asking for basic contact information. Families were eligible if they had a child aged 3-10 years old, with priority given to the youngest child if more than one child per family was eligible. Of the 691 families who completed the online interest form that asked about age, 474 were eligible to participate and were sent individual survey links to take part in the study. The reasons for exclusion were the child not being in the age range (i.e., child aged $11-18$ years old, $n=184$ ), the child being a twin and we could not discern the younger twin $(n=$ $7)$, the parent not providing a valid email address or appearing to show bot-like responses $(n=7)$, or the parent filling the interest form out twice $(n=17)$. Of the 474 families sent survey links, 349 completed the study. We subsequently excluded children reported by parents to have been diagnosed with severe a medical or genetic condition, such as epilepsy or Spina bifida $(n=8)$, Autism Spectrum Disorder $(n=7)$, or a developmental disorder, such as speech delay or specific learning impairment $(n=31)$, resulting in a final sample of 303. Surveys were presented using a secure online survey system. Informed consent was obtained electronically at the start of the survey and families were compensated with a $\$ 10$ Amazon gift card upon completion of the survey. All procedures were approved by the Institutional Review Boards at [blind for review] and [blind for review].

\section{COVID-19 Exposures and Worries}

Parents answered five questions about their exposure to COVID-19, including whether they had been tested, experienced symptoms, knew someone who tested positive, knew someone who died, and whether their job was impacted [54]. Answers for two questions were collapsed to create meaningful binary outcomes: tested for COVID-19 combined any testing (i.e., being tested and whether parents ultimately tested negative or positive) and negative impact on job combined any change in job, income, or hours (i.e., losing job, reduced hours, or reduced pay) (Table 1). Parents also reported on their level of worry for six concerns about COVID-19 (e.g., getting or dying from the virus, family getting the virus), with items rated on a 5 -point Likert scale $(1=$ not at all; $5=$ great deal; Table 1$)$ [54]. Items were combined into an overall COVID-19 worries scale $(\alpha=.83)$.

\section{Child Conduct Problems (CP)}

We assessed CP using parent report on the 5-item CP subscale of the Strengths and Difficulties Questionnaire [SDQ; 55] (e.g., "often loses temper" and "often lies or cheats), with items rated on a 3 -point scale $(0=$ not true; $2=$ 
Table 1 COVID-19 exposures and worries across the whole sample

\begin{tabular}{ll}
\hline COVID-19 exposures & $\%$ \\
\hline Tested & 12.2 \\
Symptoms attributable to COVID-19 & 13.8 \\
Know someone who tested positive & 56.6 \\
Know someone who died & 21.9 \\
Job negatively affected ${ }^{\mathrm{a}}$ & 20.3 \\
\hline COVID-19 worries ${ }^{\text {b }}$ & $M(\mathrm{SD})$ \\
\hline Family getting COVID-19 & $3.57(1.05)$ \\
Unknowingly infecting others & $3.11(1.15)$ \\
Self-getting COVID-19 & $2.91(1.02)$ \\
Financial Burden of Pandemic & $2.74(1.36)$ \\
Dying from COVID-19 & $2.26(1.13)$ \\
Currently having COVID-19 & $1.85(1.05)$ \\
\hline
\end{tabular}

${ }^{\mathrm{a}}$ Job negatively affected includes parents who reported loss of job, reduction in pay, or reduction in hours

${ }^{\mathrm{b}} \mathrm{A}$ Likert scale ranging from 1 (not at all) to 5 (a great deal) was used to assess level of worry for each of the COVID-19 worries.

certainly true). Internal consistency was adequate $(\alpha=.65)$ and comparable to estimates reported in other studies that have assessed CP via brief measures across childhood [6, 55].

\section{Child Callous-Unemotional (CU) Traits}

We assessed CU traits via parent report on the 24-item Inventory of Callous-Unemotional Traits (ICU) [56], which assesses callousness and a lack of empathy (e.g., "unconcerned about feelings of others"), uncaring and limited prosocial behavior ("always tries best"), and unemotionality ("hides feelings"), with items rated on a four-point scale ( $0=$ not true; $3=$ definitely true). Consistent with recommendations [57], we computed total ICU scores $(\alpha=.85)$.

\section{Parental Warmth}

To assess parental warmth, we used the 6-item positive involvement subscale of the Alabama Parenting Questionnaire (APQ) [58], which assesses parental use of praise, affection, and positive reinforcement (e.g., "you praise your child" and "you hug or kiss your child") with items rated on a 5 -point Likert scale $(1=$ never to $5=$ always $)$. Internal consistency for the scale was high $(\alpha=.82)$.

\section{Harsh Parenting}

To assess harsh parenting, we used the 9-item over-reactivity subscale of the Parenting Scale [59], which assesses parental use of harsh discipline, including displaying anger and irritability (e.g., "I raise my voice or yell" and "I am picky and on my child's back"), with items rated on a 1-7 scale. Internal consistency for the scale was high $(\alpha=.78)$.

\section{Covariates}

We included methodological and demographic variables as predictors or covariates as relevant within statistical models, including study site $\left(0=[\text { blind for review })^{1}, 1=[\right.$ blind for review] $)$, recruitment month $(0=$ April, $1=$ May, $2=$ June, $3=$ July $)$, parent education $(0=$ high school qualification or less, $1=$ Associates degree or higher), household income, parent race (two dummy codes for White vs. Black vs. Asian parents) and ethnicity $(0=$ non-Hispanic/Latino vs. $1=$ Hispanic/Latino), child age, sex, race (two dummy codes for White vs. Black vs. Asian children) and ethnicity $(0=$ non-Hispanic/Latino vs. $1=$ Hispanic/Latino $)$, as well as parent reports of children having a prior externalizing disorder diagnosis (i.e., oppositional defiant disorder, ODD or attention-deficit hyperactivity disorder, $\mathrm{ADHD} ; 0=$ no, $1=$ yes). Finally, we conducted additional robustness checks by running models that included parent reports of child anxiety and worry, assessed via the emotional problems subscale of the Strengths and Difficulties Questionnaire (SDQ; Goodman et al. [60] or parent reports that their child had a prior

\footnotetext{
${ }^{1}$ Demographic and study characteristics for the two sites are presented in Table S1. There were more Black and Hispanic families and fewer White families recruited in Site 2 [blind for review] than Site 1 [blind for review], more parents from Site 2 than Site 1 reported having a high school education or less, and parents from Site 2 had significantly lower monthly income and were younger in age than parents from Site 1. Site was thus included as a covariate in all study models
} 
Fig. 1 Mean level of worry reported by parents for six potential worries arising from the COVID-19 pandemic. Dotted line represents overall mean level of worry across all six worries (see Table 1). Items were rated on a 5-point Likert scale $(1=$ not at all, $2=$ a little, $3=$ moderate amount, $4=\mathrm{a}$ lot, $5=$ great deal



diagnosis of an internalizing disorder, such as depression or anxiety.

\section{Analytic Strategy}

Data were explored using regression analyses within a structural equation modeling framework in Mplus 8.1 [61]. For our first aim, we tested whether any study or family characteristics were related to parental exposures to COVID-19 or worries about COVID-19. To explore unique effects of study and family characteristics, we tested two additional models which specified the five COVID-19 exposures and the six COVID-19 worries as correlated dependent variables, with site, recruitment month, parent education, household income, parent race, parent ethnicity, and child age entered simultaneously as predictors. To address our second aim, we tested whether harsh and warm parenting were independently related to child CP or CU traits, accounting for site, recruitment month, parent education, household income, child age, sex, race, and ethnicity, and parent reports of whether children had a prior diagnosis of ADHD or ODD. To parse unique effects, we accounted for $\mathrm{CU}$ traits in the model predicting $\mathrm{CP}$ and vice versa. Third, we explored whether a cumulative sum of parental exposures to COVID-19 and parental worries about COVID-19 were independently related to child $\mathrm{CP}$ or CU traits accounting for harsh and warm parenting, as well as model covariates. In post-hoc exploratory analyses, we examined associations between $\mathrm{CP}$ and CU traits and parents' individual worries about COVID-19 (i.e., at the item- rather than scale-level) while accounting for harsh and warm parenting and model covariates, with items tested as correlated dependent variables. These results are presented in the supplementary materials. All participants were included in analyses using robust full-information maximum likelihood estimation [62]. Weighted least squares means and variance adjusted
(WLSMV) estimation was used for models with COVID-19 exposures as dependent variables to account for categorical distributions [63].

\section{Results}

Overall, parents reported significant exposure to COVID-19, including $56.6 \%$ of parents reporting personally knowing someone who had tested positive for the virus, $21.9 \%$ personally knowing someone who had died with the virus, and $20.3 \%$ reporting that they had lost their job or experienced a loss of income or hours during the pandemic (Table 1). Parents reported being most worried about a family member getting the virus, unknowingly spreading the virus, and contracting the virus themselves (Table 1 and Fig. 1). More exposure to COVID-19 (i.e., cumulative score) was correlated with greater levels of worry (Table 2).

To explore our first aim, we used regression analyses to assess predictors of COVID-19 exposures. We found that parents who were recruited to the study later were more likely to have been tested for COVID-19 $(\beta=0.35, p<.01)$, parents with younger children were more likely to report symptoms of COVID-19 $(\beta=-0.28, p<.01)$, and parents with a high school qualification or less were more likely to have experienced disruptions to their work or income due to COVID-19 $(\beta=-0.15, p<.05)$ (Table S2).

For parental worries about COVID-19, Black parents $(\beta=0.17, p<.05)$, Hispanic parents $(\beta=0.17, p<.05)$, and parents with lower monthly household incomes $(\beta$ $=-0.11, p<.05)$ reported more worry about currently having symptoms of COVID-19. Hispanic parents also reported being more worried about dying from COVID$19(\beta=0.15, p<.05)$. Finally, parents recruited in [blind for review] relative to those recruited in [blind for review] ( $\beta=0.19, p<.01)$, parents with lower monthly incomes 
Table 2 Descriptive statistics and bivariate correlations between continuous study variables

\begin{tabular}{lrlclll}
\hline & $M(\mathrm{SD})$ & Range & Exposures & Worries & Harsh parenting & Parental warmth \\
\hline COVID-19 & & & & & \\
$\quad$ Exposures & $1.25(1.10)$ & $0-5$ & & & \\
$\quad$ Worries & $16.44(4.99)$ & $6-30$ & $0.14^{*}$ & & & \\
Parenting & & & & & & \\
$\quad$ Harsh parenting & $16.64(7.49)$ & $0-41$ & -0.07 & 0.05 & -0.03 & -0.01 \\
Parental warmth & $19.53(3.05)$ & $8-24$ & 0.01 & 0.06 & & $-0.18^{* *}$ \\
Child outcomes & & & & $0.14^{*}$ & $0.27^{* *}$ & $0.50^{* *}$ \\
CP & $1.50(1.58)$ & $0-9$ & 0.01 & -0.01 & $0.36^{* *}$ & \\
CU traits & $17.07(7.73)$ & $0-53$ & 0.04 & & & \\
\hline
\end{tabular}

COVID-19 exposures was computed as a cumulative exposures score

$* p<.05$

$* * p<.01$

$* * * p<.001$

( $\beta=-0.20, p<.001)$, and parents of older children $(\beta$ $=0.13, p<.01)$ reported more worry about the lasting financial burden of the pandemic. Asian parents reported less worry about the financial burden of the pandemic $(\beta=$ $-0.15, p<.01)$ but greater worry about catching COVID$19(\beta=0.12, p<.05)$ (Table S3). Recruitment month and parent education not significantly related to worries about COVID-19.

To explore substantive associations between parental exposures to and worries about COVID-19, parenting practices, and child outcomes, we computed descriptive statistics and bivariate correlations (Table 2). To test our second aim, we explored independent associations between parenting practices and child $\mathrm{CP}$ and $\mathrm{CU}$ traits during the pandemic using regression models. Consistent with hypotheses, higher levels of harsh parenting were related to more child $\mathrm{CP}(\beta=$ $0.13, p<.01$ ) (Table 3 ) and both higher parental harshness $(\beta=0.23, p<.001)$ and lower warmth $(\beta=-0.19, p<.001)$ were independently related to higher CU traits (Table 3 ).

To test our third aim, we used regression analyses to test whether parental exposures to or worries about COVID-19 were independently related to child $\mathrm{CP}$ or CU traits, over and above parenting practices and covariates. Parent reports of COVID-19 exposures (i.e., cumulative total score) were not independently related to child CP or CU traits (Table 4). For worries, higher parental COVID-19 worry scores were uniquely related to more child $\mathrm{CP}(\beta=0.15, p<0.01)$, but not CU traits, over and above parenting practices and covariates (Table 4). In post-hoc models testing individual parent worries, we found that parental worries about themselves getting COVID-19 $(\beta=0.14, p<0.01)$ or a family member getting COVID-19 ( $\beta=0.14, p<0.01)$ were independently related to higher child CP (Table S4). There were no significant associations between individual parental worries about COVID-19 and child CU traits (Table S5).

\section{Discussion}

The COVID-19 pandemic has resulted in an unprecedent global health and financial crisis that will leave an indelible mark on this and future generations. For families, the challenges arising from the pandemic have been particularly deleterious [40]. Many parents have lost their jobs, faced reduced pay, or have had to simultaneously balance the need to earn money while taking care of and educating children [41, 42]. Children have been removed from contexts that provide educational, nutritional, and social support [40, 41], including peer interactions that help shape the development of cooperation, empathy, and emotion regulation, which are core socioemotional skills that have gone awry among children with CP and CU traits [3, 5]. Our study was motivated by the goal of understanding family and parenting experiences during the pandemic, particularly in relation to $\mathrm{CP}$ and CU traits in children, which predict long-term adverse and costly physic al and mental health outcomes [1, 2].

First, we found that more than half of parents knew someone who had been diagnosed with COVID-19 and nearly a quarter of parents knew someone that had died because of the virus. These findings are part of a growing testament to the pervasive impact that this pandemic is having on the lives of families in the US. In terms of worries about COVID-19, parents in our sample reported being most worried about a family member getting the virus, unknowingly spreading the virus, and getting the virus themselves. Our results align with a prior study of adults $(N=3042)$ recruited via online crowd-sourcing, where concerns about a family member getting the virus and unknowingly spreading the virus were similarly ranked as the top two concerns [54]. These findings are also consistent with prior evidence of increases in prosocial and "tend-and-befriend" responses during times of stress, thought to reflect an innate human 
Table 3 Harsh parenting is related to more child $\mathrm{CP}$ and $\mathrm{CU}$ traits and low parental warmth is related only to child CU traits, accounting for covariates.

\begin{tabular}{|c|c|c|c|c|c|c|}
\hline & \multicolumn{3}{|c|}{ Conduct problems } & \multicolumn{3}{|l|}{$\mathrm{CU}$ traits } \\
\hline & $\mathrm{B}$ & SE & $\beta$ & B & SE & $\beta$ \\
\hline \multicolumn{7}{|l|}{ Covariates } \\
\hline Site & 0.10 & 0.16 & 0.03 & -0.52 & 0.76 & -0.03 \\
\hline Recruitment month & 0.03 & 0.08 & 0.02 & -0.60 & 0.39 & -0.08 \\
\hline Parent education & -0.21 & 0.25 & -0.04 & 0.80 & 1.21 & 0.03 \\
\hline Household monthly income & 0.00 & 0.01 & 0.00 & 0.09 & 0.07 & 0.10 \\
\hline Child age & -0.05 & 0.04 & -0.07 & -0.17 & 0.17 & -0.05 \\
\hline Child sex & 0.15 & 0.15 & 0.05 & $1.47 *$ & 0.70 & 0.10 \\
\hline Child race (Black vs. other) & -0.22 & 0.25 & -0.05 & $2.39 *$ & 1.13 & 0.12 \\
\hline Child race (Asian vs. other) & -0.41 & 0.24 & -0.09 & 2.08 & 1.19 & 0.09 \\
\hline Child ethnicity & -0.18 & 0.24 & -0.03 & 1.35 & 1.44 & 0.05 \\
\hline Diagnosis (ODD or ADHD) & $1.98 * * *$ & 0.38 & $0.29 * * *$ & & & \\
\hline Conduct problems & & & & $2.04 * * *$ & 0.29 & $0.42 * * *$ \\
\hline CU traits & $0.09 * * *$ & 0.01 & $0.45^{* * *}$ & & & \\
\hline \multicolumn{7}{|l|}{ Parenting } \\
\hline Harsh parenting & $0.03 * *$ & 0.01 & $0.13 * *$ & $0.24 * * *$ & 0.06 & $0.23 * * *$ \\
\hline Parental warmth & 0.04 & 0.03 & 0.08 & $-0.47 * * *$ & 0.11 & $-0.19 * * *$ \\
\hline
\end{tabular}

For the model predicting $\mathrm{CU}$ traits, results were unchanged when we included parent reports of the child having a prior externalizing disorder diagnosis (i.e., ADHD or ODD) to including continuous CP scores (i.e., collinearity prevents these being included in the same model). Results for CP and CU traits were also unchanged if we included parent reports of child anxiety and worry, assessed via the emotional problems subscale of the Strengths and Difficulties Questionnaire (SDQ; Goodman et al., 2010) or if we accounted for parents reporting that children had a prior diagnosis of an internalizing disorder, such as depression or anxiety, $n=9,3 \%$ (results available on request)

$* p<.05$

$* * p<.01$

$* * * p<.001$

tendency to protect those closest to them and seek out their social group when threatened $[64,65]$, which appears to be especially the case among females and female caregivers $[54,66]$, as was the case for the vast majority of parents in our sample.

Consistent with a growing literature [67-70], we found that the pandemic is not affecting all families equally. In particular, despite a relatively small proportion of non-White parents in our sample, we found racial and ethnic disparities in parental exposures to and concerns about COVID19. Hispanic parents reported more worry about dying from COVID-19 and both Black and Hispanic parents reported being more worried about currently having symptoms of COVID-19. These effects emerged in stringent multivariate models that included the effects of lower monthly household income, site, and child age. In addition, we found that lower education was a unique predictor of whether parents reported experiencing a disruption to their job or income due to COVID-19. Thus, when combined with the results for worries, our results provide further support for the disproportionate health, psychological, and financial burden that the pandemic is placing on Black and Hispanic families, as well as parents without higher educational qualifications who may hold jobs that are more vulnerable to the economic fallout of the pandemic [67-70]. As we move towards recovery, and even as the pandemic continues to wreak havoc on the daily routines and lives of families, it remains imperative that relief efforts are targeted towards segments of the population most in need.

Second, consistent with hypotheses and extant literature, higher levels of harsh parenting, including more overreactive and punitive forms of discipline reported by parents, were related both to more child $\mathrm{CP}$ and higher $\mathrm{CU}$ traits. Findings are consistent with a large literature on harsh parenting, which exacerbates coercive and negative parent-child interactions, leading to an escalation in non-compliant, aggressive, and disruptive child behavior, further increasing risk for CP and CU traits [12-15]. At the same time, via potentially distinct etiological mechanisms, exposure to harsh discipline can desensitize children who are relatively more fearless to both punishment and the negative consequences of their behavior, thereby increasing risk for $\mathrm{CU}$ traits [22, 23, 71]. Consistent with prior studies, we also found a unique effect of lower parental warmth on child CU traits, but not CP [23, 25]. Our findings support the assertion that fewer experiences of rewarding affiliative interactions with 
Table 4 Parental worries about COVID-19 are independently related to child $\mathrm{CP}$, but not $\mathrm{CU}$ traits, accounting for parenting, COVID-19 exposures, and covariates

\begin{tabular}{|c|c|c|c|c|c|c|}
\hline & \multicolumn{3}{|c|}{ Conduct Problems } & \multicolumn{3}{|l|}{ CU traits } \\
\hline & $\mathrm{B}$ & SE & $\beta$ & B & SE & $\beta$ \\
\hline \multicolumn{7}{|l|}{ Covariates } \\
\hline Site & 0.08 & 0.16 & 0.03 & -0.50 & 0.77 & -0.03 \\
\hline Recruitment month & 0.02 & 0.08 & 0.01 & -0.56 & 0.38 & -0.07 \\
\hline Parent education & -0.20 & 0.24 & -0.04 & 0.80 & 1.22 & 0.03 \\
\hline Household monthly income & 0.00 & 0.01 & 0.02 & 0.08 & 0.07 & 0.08 \\
\hline Child age & -0.05 & 0.04 & -0.07 & -0.18 & 0.17 & -0.05 \\
\hline Child sex & 0.16 & 0.15 & 0.05 & 1.48 & 0.70 & $0.10^{*}$ \\
\hline Child race (Black vs. other) & -0.25 & 0.25 & -0.06 & 2.36 & 1.13 & $0.12 *$ \\
\hline Child race (Asian vs. other) & -0.46 & 0.24 & -0.10 & 2.37 & 1.19 & $0.10 *$ \\
\hline Child ethnicity & -0.23 & 0.24 & -0.04 & 1.49 & 1.48 & 0.06 \\
\hline Diagnosis (ODD or ADHD) & $2.01 * * *$ & 0.36 & $0.29 * * *$ & & & \\
\hline Conduct problems & & & & 2.08 & 0.29 & $0.43 * * *$ \\
\hline CU traits & $0.09 * * *$ & 0.02 & $0.45 * * *$ & & & \\
\hline \multicolumn{7}{|l|}{ Parenting } \\
\hline Harsh parenting & $0.02 *$ & 0.01 & $0.12 *$ & $0.25 * * *$ & 0.05 & $0.24 * * *$ \\
\hline Parental warmth & 0.04 & 0.03 & 0.08 & $-0.47 * * *$ & 0.11 & $-0.19 * * *$ \\
\hline \multicolumn{7}{|l|}{ COVID-19 } \\
\hline Exposures & -0.08 & 0.07 & -0.06 & 0.57 & 0.36 & 0.08 \\
\hline Worries & $0.05^{* *}$ & 0.02 & $0.15 * *$ & -0.11 & 0.07 & -0.07 \\
\hline
\end{tabular}

For the model predicting CU traits, results were unchanged when we included parent reports of the child having a prior externalizing disorder diagnosis (i.e., ADHD or ODD) to including continuous CP scores (i.e., collinearity prevents these being included in the same model). Results for CP and CU traits were also unchanged if we included parent reports of child anxiety and worry, assessed via the emotional problems subscale of the Strengths and Difficulties Questionnaire (SDQ; Goodman et al., 2010) or if we accounted for parents reporting that children had a prior diagnosis of an internalizing disorder, such as depression or anxiety, $n=9,3 \%$ (results available on request)

$* p<.05$
$* * p<.01$
$* * * p<.001$

a parent (i.e., warm and affectionate parental inputs), may provide a child with less opportunity to mimic, practice, or develop behaviors that promote social bonding, cooperation, and affiliation with others, thus increasing risk for $\mathrm{CU}$ traits $[20,71]$. The reported pattern of findings was unchanged when parents' worries about and exposures to COVID-19 were included as predictors, highlighting the robust associations between parenting practices and both child CP and CU traits.

Finally, we tested whether children were directly affected by the level of family exposure to or worry about the pandemic, and importantly, whether the pandemic was impacting $\mathrm{CP}$ or CU traits over and above the influence of parenting practices. We found that greater levels of parental worry about the pandemic, particularly worries about themselves or family members getting the virus, were uniquely related to more child $\mathrm{CP}$, but not child CU traits. This finding emphasizes that distinct risk factors appear to be associated with different pathways to $\mathrm{CP}$ and CU traits. That is, existing research suggests that among children with relatively greater levels of negative reactivity and emotion dysregulation (i.e., low CU traits), exposure to parental worry may further exacerbate risk for $\mathrm{CP}$ [71]. In contrast, children who are more fearless and insensitive to negative emotions (i.e., those with high CU traits), may be relatively more impervious to the stressors facing families [20, 71]. Of note, there were no direct effects from any of the parental exposures to COVID19 on $\mathrm{CP}$, only from parental worries. One possibility is that children may not have been told about nor be aware of those specific exposures (i.e., changes in job/hours, parent personally knowing someone who contracted the virus or died with the virus). However, children would pick up on and be aware of stress exhibited by their parents that could arise from worries about COVID-19. Indeed, parents experiencing higher levels of stress because of exposure to or concerns about the pandemic may confer unmeasured disruptions to family dynamics, routines, and interactions within the family in ways that specifically impact children's likelihood of noncompliance, aggression, or disruptive behavior, [32, 38, 39]. 
However, contrary to hypotheses and somewhat surprisingly, exposure to and worries about the pandemic were not significantly correlated with parenting practices, which stands at odds with research linking contextual stress and low social support to harsher and less warm parenting [28, $40,44]$. At the same time, there is evidence for moderate stability in parenting practices, particularly in sensitive parenting behaviors, across early and late childhood [72, 73]. One possibility is that some parents were able to adapt their parenting practices to be more warm and less harsh, acutely aware of the potential deleterious effects of the pandemic on their children (i.e., adaptive buffering or protective effect) $[74,75]$. However, given the chronic nature of the pandemic, another possibility is that the impact of familial exposure and worry about COVID-19 may also instead exert a delayed effect on parenting. Longitudinal studies that continue to follow families are needed to chart the evolving effects of COVID-19 on family life and parenting. Nevertheless, our results highlight the continued need for broad efforts to mitigate the negative consequences of the pandemic that focus on family systems and parenting, as well as directly addressing children's perceptions and fears about COVID-19 that might arise from family exposure and parental worries [48, 76].

The current findings should be considered in the context of several limitations. First, our survey was necessarily brief to limit burden on parents. Thus, other negative consequences of the pandemic (e.g., social isolation, parental mental health) and their effects on parenting and child outcomes were not assessed. Second, this was a crosssectional study, which prohibits strong causal conclusions. Moreover, we did not have data on parenting practices and child CU traits and CP prior to the pandemic. However, the current findings serve as an important foundation for future research to explore changes in parenting and child CU traits and $\mathrm{CP}$ as the pandemic continues to disrupt the lives of families. Third, the internal consistency of our measure of $\mathrm{CP}$ was lower than estimates that are typically considered as indicative of good reliability. Fourth, while there was a wide range in family income in our sample, the median monthly household income was above that for the cities from which parents were recruited and levels of education of our sample were skewed towards parents being more highly educated. Thus, our findings may not generalize to samples including more parents without college degrees or among families with monthly household incomes falling below the median incomes in [blind for review] and [blind for review]. Fifth, while there were differences in the exposures to and worries about COVID-19 based on race and ethnicity, these analyses were limited because the majority of our sample were White. Because of our relatively smaller sample size, we also assigned multiracial Black and White parents/children to the Black subsample and biracial Asian and White parents/ children to the Asian subsample. This decision may be warranted given that historically in the US, part-White multiracial individuals have been treated through the lens of their minority racial identity [77], including in the experience of discrimination [78]. However, multiracial individuals also face many specific challenges, including racial identity invalidation [79] and incongruent discrimination [80], which may amount to a qualitatively different experiences than those of individuals who identify as belonging to a single race. Future studies with larger sample sizes are needed to explore differences in exposure to and worries about COVID-19, as well as long-term outcomes of the pandemic, among multiracial individuals. Sixth, we did not include measures to assess home schooling experiences in this survey, which was an unfortunate oversight given that for parents, working from home while simultaneously managing the full-time "homeschooling" or virtual learning experiences of their children has represented a significant source of stress [75]. Follow-up data collected from this cohort does include such measures, thus future studies will thus be able to address how stress arising from home-schooling/virtual learning has impacted families. Finally, given the stay-at-home orders at the time of data collection, we necessarily relied on parent report for all measures, which may have artificially inflated documented associations due to shared method variance.

In sum, our findings provide further empirical evidence documenting the unprecedent harm that the pandemic is inflicting on families, particularly Black and Hispanic parents in our sample. Moreover, we add to a growing literature showing that specific parenting practices are differentially related to $\mathrm{CP}$ and $\mathrm{CU}$ traits, which can inform the development of personalized, adjunctive modules for parenting interventions for childhood CP [26, 27]. Our results address several commentaries from the start of the pandemic, which emphasized that the insidious combination of factors associated with the pandemic might increase the risk for child abuse and an escalation in violence exposure [40, 43]. Future follow-up studies in this cohort can document how the pandemic might contribute to changes in family and parenting dynamics. At the same time, the pandemic has galvanized researchers and practitioners towards online interventions to promote effective parenting [81], which may be particularly helpful to reduce child CP and CU traits. Indeed, a recent meta-analysis of 12 studies found that online parenting programs are effective in reducing child CP and improving parental mental health problems [82]. The pandemic has placed unprecedented burden and stress on families across the globe, but it has also shown us that many barriers to mental health services can be overcome, paving the way to make such efforts universally accessible in the future, including via telehealth services for traditionally hard-to-reach families $[83,84]$. 


\section{Summary}

Since January 2020, the COVID-19 pandemic has dramatically altered family life, upending normal routines and social interactions. However, whether family exposures to and worries about the COVID-19 pandemic has impacted child CP and $\mathrm{CU}$ traits, beyond the influence of parenting practices, is unknown. The current study addressed this knowledge gap by examining the associations between exposures to COVID-19, parental worries about the pandemic, harsh and warm parenting practices, and child $\mathrm{CP}$ and $\mathrm{CU}$ traits. We find that black and Hispanic parents, as well as parents with lower monthly household incomes, reported more exposure to and concerns about COVID-19. Higher levels of parental harshness were related to higher levels of child $\mathrm{CP}$ and $\mathrm{CU}$ traits, while lower levels of parental warmth were uniquely related to higher CU traits. Although more parental worries were not related to parenting practices, more parental worry about COVID-19 was specifically related to higher levels of child CP, particularly parental worries about themselves or family members contracting the virus. Our findings add to a growing literature demonstrating the enormous burden that the pandemic has placed on families. The results also speak to the continued importance of targeting parenting practices to reduce child $\mathrm{CP}$ and $\mathrm{CU}$ traits even as families contend with the continued, pervasive stress imbued by the pandemic.

Acknowledgements This work was funded by institutional support from the University of Pennsylvania (RW), the John and Polly Sparks Early Career Grant from the American Psychological Foundation (RW), institutional support from Boston University (NJW), and the National Institute of Mental Health (NIMH), Grant K23 MH120437 (RB).

\section{Compliance with Ethical Standards}

Conflict of interest The authors report no conflicts of interest.

\section{References}

1. Foster EM, Jones DE, Group CPPR (2005) The high costs of aggression: Public expenditures resulting from conduct disorder. Am J Public Health 95(10): 1767-1772

2. Rivenbark JG, Odgers CL, Caspi A, Harrington H, Hogan S, Houts RM, Poulton R, Moffitt TE (2018) The high societal costs of childhood conduct problems: evidence from administrative records up to age 38 in a longitudinal birth cohort. J Child Psych Psychiatry 59(6):703-710

3. Waller R, Wagner NJ, Barstead MG, Subar A, Petersen JL, Hyde JS, Hyde LW (2020) A meta-analysis of the associations between callous-unemotional traits and empathy, prosociality, and guilt. Clin Psychol Rev 75:101809

4. Longman T, Hawes DJ, Kohlhoff J (2016) Callous-unemotional traits as markers for conduct problem severity in early childhood: a meta-analysis. Child Psychiatry Human Dev 47(2):326-334

5. Waller R, Hyde LW (2018) Callous-unemotional behaviors in early childhood: the development of empathy and prosociality gone awry. Curr Opin Psychol 20:11-16

6. Willoughby MT, Mills-Koonce WR, Gottfredson NC, Wagner NJ (2014) Measuring callous unemotional behaviors in early childhood: factor structure and the prediction of stable aggression in middle childhood. J Psychopathol Behav Asses 36(1):30-42

7. Cardinale EM, Marsh AA (2020) The reliability and validity of the inventory of callous unemotional traits: a meta-analytic review. Assessment 27(1):57-71

8. McMahon RJ, Witkiewitz K, Kotler JS (2010) Predictive validity of callous-unemotional traits measured in early adolescence with respect to multiple antisocial outcomes. J Abnormal Psychol 119(4):752

9. Association AP (2013) Diagnostic and statistical manual of mental disorders (DSM-5®): Am Psychiatr Pub

10. Hyde LW, Waller R, Burt SA (2014) Commentary: improving treatment for youth with callous-unemotional traits through the intersection of basic and applied science-reflections on Dadds, et al 2014. J Child Psychol Psychiatry 55(7):781-783

11. Burt SA (2009) Rethinking environmental contributions to child and adolescent psychopathology: a meta-analysis of shared environmental influences. Psychol Bull 135(4):608

12. Shaw DS, Galán CA, Lemery-Chalfant K, Dishion TJ, Elam KK, Wilson MN, Gardner F (2019) Trajectories and predictors of children's early-starting conduct problems: child, family, genetic, and intervention effects. Dev Psychopathol 31(5):1911-1921

13. Sitnick SL, Shaw DS, Weaver CM, Shelleby EC, Choe DE, Reuben JD, Gilliam M, Winslow EB, Taraban L (2017) Early childhood predictors of severe youth violence in low-income male adolescents. Child Dev 88(1):27-40

14. Dodge KA, Bates JE, Pettit GS (1990) Mechanisms in the cycle of violence. Science 250(4988):1678-1683

15. Dishion TJ, Patterson GR, Kavanagh KA (1992) An experimental test of the coercion model: linking theory, measurement, and intervention: Guilford Press

16. Gardner F, Shaw DS, Dishion TJ, Burton J, Supplee L (2007) Randomized prevention trial for early conduct problems: effects on proactive parenting and links to toddler disruptive behavior. J Fam Psychol 21(3):398

17. Leijten P, Gardner F, Landau S, Harris V, Mann J, Hutchings J, Beecham J, Bonin EM, Scott S (2018) Research review: harnessing the power of individual participant data in a meta-analysis of the benefits and harms of the Incredible Years parenting program. J Child Psychol Psychiatry 59(2):99-109

18. Wootton JM, Frick PJ, Shelton KK, Silverthorn P (1997) Ineffective parenting and childhood conduct problems: the moderating role of callous-unemotional traits. J Consult Clin Psychol 65(2):301

19. Moore AA, Blair RJ, Hettema JM, Roberson-Nay R (2019) The genetic underpinnings of callous-unemotional traits: a systematic research review. Neurosci Biobehav Rev 100:85-97

20. Waller R, Wagner N (2019) The sensitivity to threat and affiliative reward (STAR) model and the development of callous-unemotional traits. Neurosci Biobehavl Rev 107:656-671

21. Waller R, Trentacosta CJ, Shaw DS, Neiderhiser JM, Ganiban JM, Reiss D, Leve LD, Hyde LW (2016) Heritable temperament pathways to early callous-unemotional behaviour. Br J Psychiatry 209(6):475-482

22. Trentacosta CJ, Waller R, Neiderhiser JM, Shaw DS, Natsuaki MN, Ganiban JM, Reiss D, Leve LD, Hyde LW (2019) Callousunemotional behaviors and harsh parenting: reciprocal associations across early childhood and moderation by inherited risk. J Abnormal Child Psychol 47(5):811-823 
23. Waller R, Hyde LW, Klump KL, Burt SA (2018) Parenting is an environmental predictor of callous-unemotional traits and aggression: a monozygotic twin differences study. J Am Acad Child Adolesc Psychiatry 57(12):955-963

24. Clark JE, Frick PJ (2018) Positive parenting and callousunemotional traits: their association with school behavior problems in young children. J Clin Child Adolesc Psychol 47(sup1):S242-S254

25. Henry J, Dionne G, Viding E, Vitaro F, Brendgen M, Tremblay RE, Boivin M (2018) Early warm-rewarding parenting moderates the genetic contributions to callous-unemotional traits in childhood. J Child Psychol Psychiatry 59(12):1282-1288

26. Kimonis ER, Fleming G, Briggs N, Brouwer-French L, Frick PJ, Hawes DJ, Bagner DM, Thomas R, Dadds M (2019) Parent-child interaction therapy adapted for preschoolers with callous-unemotional traits: an open trial pilot study. J Clin Child Adolesc Psychol 48(sup1):S347-S361

27. Dadds MR, English T, Wimalaweera S, Schollar-Root O, Hawes DJ (2019) Can reciprocated parent-child eye gaze and emotional engagement enhance treatment for children with conduct problems and callous-unemotional traits: a proof-of-concept trial. J Child Psychol Psychiatry 60(6):676-685

28. Belsky J (1984) The determinants of parenting: a process model. Child development 83-96.

29. Mills-Koonce WR, Willoughby MT, Garrett-Peters P, Wagner $\mathrm{N}$, Vernon-Feagans L, Investigators FLPK (2016) The interplay among socioeconomic status, household chaos, and parenting in the prediction of child conduct problems and callous-unemotional behaviors. Dev Psychopathol 28(3):757-771

30. Lahey BB, Van Hulle CA, Rathouz PJ, Rodgers JL, D'Onofrio BM, Waldman ID (2009) Are oppositional-defiant and hyperactive-inattentive symptoms developmental precursors to conduct problems in late childhood?: Genetic and environmental links. J Abnormal Child Psychol 37(1):45-58

31. Bronfenbrenner U, Ceci SJ (1994) Nature-nuture reconceptualized in developmental perspective: a bioecological model. Psychol Rev 101(4):568

32. Masarik AS, Conger RD (2017) Stress and child development: a review of the family stress model. Curr Opin Psychol 13:85-90

33. Shaw DS, Shelleby EC (2014) Early-starting conduct problems: intersection of conduct problems and poverty. Ann Rev Clin Psychol 10:503-528

34. Costello EJ, Compton SN, Keeler G, Angold A (2003) Relationships between poverty and psychopathology: a natural experiment. Jama 290(15):2023-2029

35. Gennetian LA, Miller C (2002) Children and welfare reform: a view from an experimental welfare program in Minnesota. Child Dev 73(2):601-620

36. Piotrowska PJ, Stride CB, Croft SE, Rowe R (2015) Socioeconomic status and antisocial behaviour among children and adolescents: a systematic review and meta-analysis. Clin Psychol Rev $35: 47-55$

37. Wagner NJ, Mills-Koonce WR, Willoughby MT, Cox MJ (2017) Parenting and cortisol in infancy interactively predict conduct problems and callous-unemotional behaviors in childhood. Child Dev 00:1-19. https://doi.org/10.1111/cdev.12900

38. Waller R, Shaw DS, Forbes EE, Hyde LW (2015) Understanding early contextual and parental risk factors for the development of limited prosocial emotions. J Abnorm Child Psychol 43(6):1025-1039

39. Jocson RM, McLoyd VC (2015) Neighborhood and housing disorder, parenting, and youth adjustment in low-income urban families. Am J Commun Psychol 55(3-4):304-313

40. Cluver L, Lachman JM, Sherr L, Wessels I, Krug E, Rakotomalala S, Blight S, Hillis S, Bachmand G, Green O (2020) Parenting in a time of COVID-19
41. Van Lancker W, Parolin Z (2020) COVID-19, school closures, and child poverty: a social crisis in the making. Lancet Public Health 5(5): $243-\mathrm{e} 244$

42. Kalenkoski CM, Pabilonia SW (2020) Initial impact of the COVID-19 pandemic on the employment and hours of selfemployed coupled and single workers by gender and parental status

43. Coyne LW, Gould ER, Grimaldi M, Wilson KG, Baffuto G, Biglan A (2020) First things first: parent psychological flexibility and self-compassion during COVID-19. Behav Anal Pract 1.

44. Beckerman M, van Berkel SR, Mesman J, Alink LR (2017) The role of negative parental attributions in the associations between daily stressors, maltreatment history, and harsh and abusive discipline. Child Abuse Neglect 64:109-116

45. Gard AM, Waller R, Shaw DS, Forbes EE, Hariri AR, Hyde LW (2017) The long reach of early adversity: parenting, stress, and neural pathways to antisocial behavior in adulthood. Biol Psychiatry: Cognit Neurosci Neuroimaging 2(7):582-590

46. Brown SM, Doom J, Watamura SE, Lechuga-Pena S, Koppels $\mathrm{T}$ (2020) Stress and parenting during the Global COVID-19 pandemic

47. Fite PJ, Greening L, Stoppelbein L (2008) Relation between parenting stress and psychopathic traits among children. Behav Scie Law 26(2):239-248

48. Barlett J, Griffin J, Thomson D (2020) Resources for supporting children's emotional well-being during the COVID-19 pandemic. In: Recuperado de https://www.childtrends.org/publications/resou rces-for

49. Mouratidi P-S, Bonoti F, Leondari A (2016) Children's perceptions of illness and health: an analysis of drawings. Health Educat J 75(4):434-447

50. Slaughter V, Griffiths M (2007) Death understanding and fear of death in young children. Clin Child Psychol Psychiatry 12(4):525-535

51. Sprang G, Silman M (2013) Posttraumatic stress disorder in parents and youth after health-related disasters. Disaster Med Public Health Prep 7(1):105-110

52. Osofsky HJ, Osofsky JD, Kronenberg M, Brennan A, Hansel TC (2009) Posttraumatic stress symptoms in children after Hurricane Katrina: predicting the need for mental health services. Am J Orthopsychiatry 79(2):212-220

53. Stanke C, Murray V, Amlôt R, Nurse J, Williams R (2012) The effects of flooding on mental health: outcomes and recommendations from a review of the literature. PLoS curr 4.

54. Barzilay R, Moore TM, Greenberg DM, DiDomenico GE, Brown LA, White LK, Gur RC, Gur RE (2020) Resilience, COVID19-related stress, anxiety and depression during the pandemic in a large population enriched for healthcare providers. Transl Psychiatry 10(1):1-8

55. Waller R, Shaw DS, Neiderhiser JM, Ganiban JM, Natsuaki MN, Reiss D, Trentacosta CJ, Leve LD, Hyde LW (2017) Toward an understanding of the role of the environment in the development of early callous behavior. J Personal 85(1):90-103

56. Frick PJ (2004) The inventory of callous-unemotional traits. Unpublished rating scale

57. Ray JV, Frick PJ (2018) Assessing callous-unemotional traits using the total score from the inventory of callous-unemotional traits: A meta-analysis. J Clin Child Adolesc Psychol

58. Essau CA, Sasagawa S, Frick PJ (2006) Psychometric properties of the Alabama parenting questionnaire. J Child Fam Stud 15(5):595-614

59. Arnold DS, O'leary SG, Wolff LS, Acker MM (1993) The Parenting Scale: a measure of dysfunctional parenting in discipline situations. Psychol Assess 5(2):137 
60. Goodman R (2001) Psychometric properties of the strengths and difficulties questionnaire. J Am Acad Child Adolesc Psychiatry 40(11):1337-1345

61. Muthén L, Muthén B (2018) Mplus. The comprehensive modelling program for applied researchers: user's guide 5

62. Enders CK, Bandalos DL (2001) The relative performance of full information maximum likelihood estimation for missing data in structural equation models. Struct Equ Model 8(3):430-457

63. Muthén B, Asparouhov T (2002) Latent variable analysis with categorical outcomes: Multiple-group and growth modeling in Mplus. Mplus web notes 4(5):1-22

64. Buchanan TW, Preston SD (2014) Stress leads to prosocial action in immediate need situations. Front Behav Neurosci 8:5

65. Taylor SE, Klein LC, Lewis BP, Gruenewald TL, Gurung RA, Updegraff JA (2000) Biobehavioral responses to stress in females: tend-and-befriend, not fight-or-flight. Psychol Rev 107(3):411

66. Levy KN, Hlay JK, Johnson BN, Witmer CP (2019) An attachment theoretical perspective on tend-and-befriend stress reactions. Evolut Psychol Sci 5(4):426-439

67. Tai DBG, Shah A, Doubeni CA, Sia IG, Wieland ML (2020) The disproportionate impact of COVID-19 on racial and ethnic minorities in the United States. Clin Infect Dis

68. Yancy CW (2020) COVID-19 and African Americans. JAMA 33:1891-1892

69. Galea S, Abdalla SM (2020) COVID-19 Pandemic, Unemployment, and Civil Unrest: Underlying Deep Racial and Socioeconomic Divides. JAMA

70. Poteat T, Millett G, Nelson LE, Beyrer C (2020) Understanding COVID-19 risks and vulnerabilities among black communities in america: the lethal force of syndemics. Ann Epidemiol

71. Waller R, Wagner NJ, Flom M, Ganiban J, Saudino KJ (2019) Fearlessness and low social affiliation as unique developmental precursors of callous-unemotional behaviors in preschoolers. Psychol Med 1-9

72. Dallaire DH, Weinraub M (2005) The stability of parenting behaviors over the first 6 years of life. Early Childhood Res Q 20(2):201-219

73. Else-Quest NM, Clark R, Tresch Owen M (2011) Stability in mother-child interactions from infancy through adolescence. Parenting 11(4):280-287

74. Ellis BJ, Bianchi J, Griskevicius V, Frankenhuis WE (2017) Beyond risk and protective factors: an adaptation-based approach to resilience. Perspect Psychol Sci 12(4):561-587
75. Lee SJ, Ward KP, Chang OD, Downing KM (2020) Parenting Activities and the Transition to Home-based Education During the COVID-19 Pandemic. Children Youth Serv Rev 10558

76. Dalton L, Rapa E, Stein A (2020) Protecting the psychological health of children through effective communication about COVID-19. Lancet Child Adolesc Health 4(5):346-347

77. Ho AK, Sidanius J, Levin DT, Banaji MR (2011) Evidence for hypodescent and racial hierarchy in the categorization and perception of biracial individuals. J Personal Soc Psychol 100(3):492

78. Giamo LS, Schmitt MT, Outten HR (2012) Perceived discrimination, group identification, and life satisfaction among multiracial people: a test of the rejection-identification model. Cult Divers Ethnic Minor Psychol 18(4):319

79. Lou E, Lalonde RN, Wilson C (2011) Examining a multidimensional framework of racial identity across different biracial groups. Asian Am J Psychol 2(2):79

80. Franco M (2019) Let the racism tell you who your friends are: The effects of racism on social connections and life-satisfaction for Multiracial people. Int J Intercult Relat 69:54-65

81. James Riegler L, Raj SP, Moscato EL, Narad ME, Kincaid A, Wade SL (2020) Pilot trial of a telepsychotherapy parenting skills intervention for veteran families: Implications for managing parenting stress during COVID-19. J Psychother Integrat 30(2):290

82. Thongseiratch T, Leijten P, Melendez-Torres G (2020) Online parent programs for children's behavioral problems: a meta-analytic review. Eur Child Adoles Psychiatry 1-14

83. Gurwitch RH, Salem H, Nelson MM, Comer JS (2020) Leveraging parent-child interaction therapy and telehealth capacities to address the unique needs of young children during the COVID19 public health crisis. Theory, Research, Practice, and Policy, Psychological Trauma

84. Falicov C, Niño A, D’Urso MS (2020) Expanding possibilities: flexibility and solidarity with under resourced immigrant families during the Covid-19 pandemic. Fam Process

Publisher's Note Springer Nature remains neutral with regard to jurisdictional claims in published maps and institutional affiliations. 